\title{
EFFECTS OF ELECTROMAGNETIC PULSES ON A MULTILAYERED SYSTEM
}

\author{
A. Upia, K. M. Burke, J. L. Zirnheld \\ Energy Systems Institute, Department of Electrical Engineering, \\ University at Buffalo, 230 Davis Hall, Buffalo, NY 14260 \\ H. L. Moore, P. Haney \\ US Army ARDEC, AMSRD-AAR-MEM, Building 65 S, Picatinny Arsenal, NJ 07806
}

\section{Abstract}

Electromagnetic pulses (EMP) can be very destructive and protecting against them is crucial. This paper discusses a method to determine the effects an intentional EMP would have on a system with multiple layers of different materials by simulating the exposure to electromagnetic waves in ANSYS HFSS. Worst case scenarios are tested by applying wavelengths that result in a resonant frequency through the object. The results show the magnitude of the current densities at the surface of the object induced by the EMP waves. The impact of the waves can be determined by reviewing the current density through the object with respect to the physical properties of the materials.

\section{Electromagnetic Pulse}

There are a few types of EMPs that are of concern regarding electronics and system infrastructures; they are high-altitude EMP (HEMP) generated from nuclear detonation in space, and EMP and REMP which can be non-nuclear and generated from several sources. The focus is on non-nuclear EMP generated by repeatable high-power generators. Repetitive EMP (REMP) is usually wideband with each pulse being composed of a wide range of frequencies. This larger spectrum of a wideband REMP has a higher chance of striking a resonant frequency than a narrowband attack. Even though REMP tend to have a weaker field, hitting a resonant frequency can have very impactful results. [1]

\section{Simulation SETUP}

The simulations were conducted in ANSYS HFSS 13, which is for 3-D full-wave electromagnetic field simulation and high frequency and high speed components [2]. The model used was designed and imported from Autodesk Inventor.

It is being assumed that an EMP will propagate in free space as a plane wave because the distance from the source of radiation is not a factor in these simulations and a plane wave will cover both far and short distance waves [3]. A vacuum box was designed around the model to eliminate any external interference of the pulses. The incident plane wave that was selected was at the low end of the possible range of magnitudes that wideband waveforms usually are in. Beginning at the lower end of the spectrum would allow for the focus on resonant frequency as opposed to the power of the waves.

Considering the size of the system, the frequency range that was considered was from $1 \mathrm{GHz}$ to 101 $\mathrm{GHz}$, due to the belief that resonating frequencies will be in this range.

\section{MODEL}

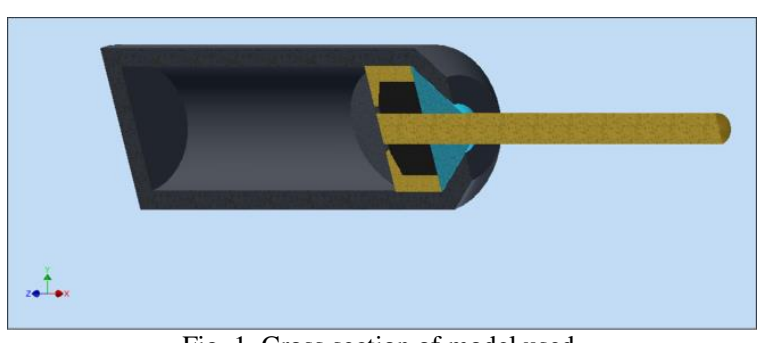

Fig. 1. Cross section of model used.

The object used for modeling was designed to be a system of components of different shapes and materials layered within each other to view how the waves would penetrate and propagate or couple to the model shown in Fig. 1, which shows the cross section with the objects differentiated by color as follows:

- The outer object (dark grey) is a steel cylinder with a small opening on the right side of the figure.

- Inside the cylinder is a metal ring (yellow) that is in direct contact with the cylinder.

- Underneath this metal is glass (black) separating the ring from the rod.

- The metal rod (yellow), which extends out through the opening, was designed to

Distribution A/Approved for Public Release - PAO Log \# 122-14-25 FEB 2014/Distribution Unlimited 


\section{Report Documentation Page}

Public reporting burden for the collection of information is estimated to average 1 hour per response, including the time for reviewing instructions, searching existing data sources, gathering and maintaining the data needed, and completing and reviewing the collection of information. Send comments regarding this burden estimate or any other aspect of this collection of information, including suggestions for reducing this burden, to Washington Headquarters Services, Directorate for Information Operations and Reports, 1215 Jefferson Davis Highway, Suite 1204, Arlington VA 22202-4302. Respondents should be aware that notwithstanding any other provision of law, no person shall be subject to a penalty for failing to comply with a collection of information if it does not display a currently valid OMB control number.

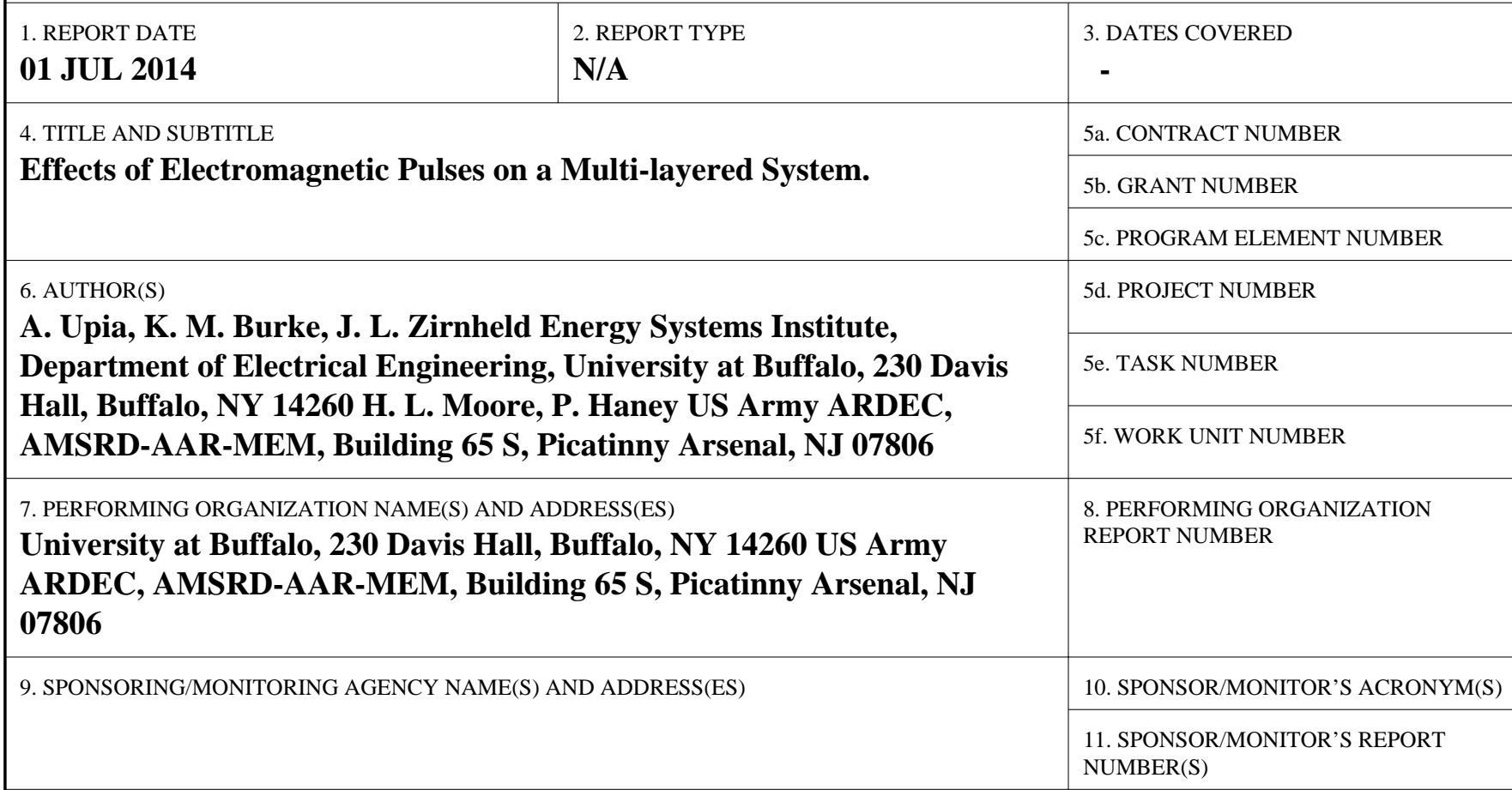

12. DISTRIBUTION/AVAILABILITY STATEMENT

Approved for public release, distribution unlimited

13. SUPPLEMENTARY NOTES

The original document contains color images.

14. ABSTRACT

Electromagnetic pulses (EMP) can be very destructive and protecting against them is crucial. This paper discusses a method to determine the effects an intentional EMP would have on a system with multiple layers of different materials by simulating the exposure to electromagnetic waves in ANSYS HFSS. Worst case scenarios are tested by applying wavelengths that result in a resonant frequency through the object. The results show the magnitude of the current densities at the surface of the object induced by the EMP waves. The impact of the waves can be determined by reviewing the current density through the object with respect to the physical properties of the materials.

15. SUBJECT TERMS

EMP, Electromagnetic Pulse

\begin{tabular}{|c|c|c|c|c|c|}
\hline \multicolumn{2}{|l|}{ 16. SECURITY CLASSIFICATION OF: } & $\begin{array}{c}\text { 17. LIMITATION OF } \\
\text { ABSTRACT } \\
\text { SAR }\end{array}$ & $\begin{array}{c}\text { 18. NUMBER } \\
\text { OF PAGES }\end{array}$ & $\begin{array}{c}\text { 19a. NAME OF } \\
\text { RESPONSIBLE PERSON }\end{array}$ \\
$\begin{array}{c}\text { unclassified } \\
\text { unclart }\end{array}$ & $\begin{array}{c}\text { b. ABSTRACT } \\
\text { unclassified }\end{array}$ & $\begin{array}{c}\text { c. THIS PAGE } \\
\text { unclassified }\end{array}$ & SAR & \\
\end{tabular}




\section{UNCLASSIFIED}

determine the effectiveness of the wave if it resonated an extension of the object.

- Although physically separated, the rod and ring are connected by a small piece of wire too small to see in the figure.

- The opening of the cylinder is sealed with silicon (blue).

The EMP is travelling from directly above the cross section in the y-direction.

\section{Simulation Results}

The following simulation results are shown at $1 \mathrm{GHz}, 11 \mathrm{GHz}$, and $31 \mathrm{GHz}$ and are grouped in order by frequency of impinging wave. Each grouping consists of 3 points of interest in the model, E Field at the end of the external rod, current density at the internal end of the rod where multiple materials come together including a fine gauge wire, and current density at the surface of the wire that connects the rod and ring.

Grouping one, comprised of figures 2, 3 and 4, show the results of the model bombarded with a wave at $1 \mathrm{GHz}$. This shows that the E field is strongest at the tip of the rod away from the other components, and that there is no appreciable concentration of the current density at the surface of the rod internal to the cylinder and the wire connecting the rod and the ring.

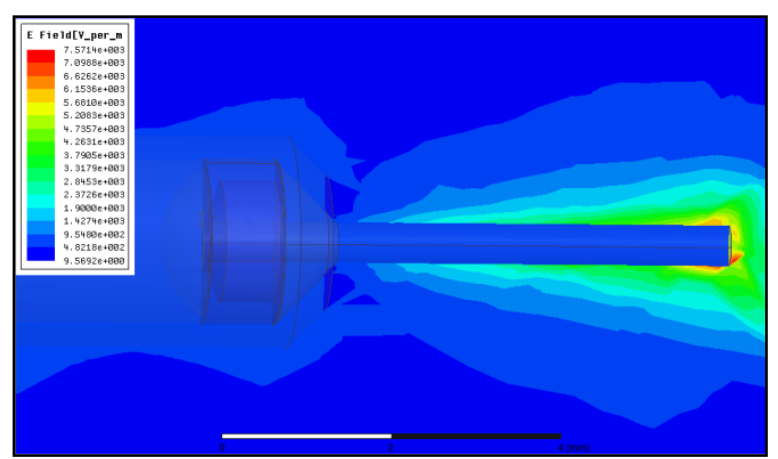

Fig. 2. E field results of $1 \mathrm{GHz}$ wave

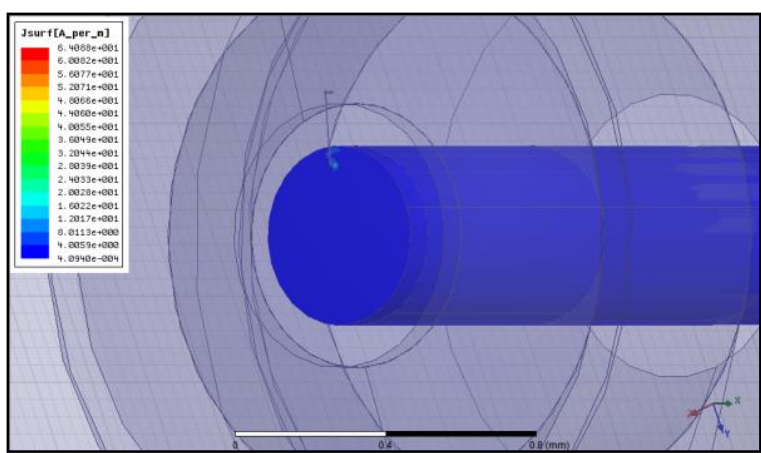

Fig. 3. Current density at surface of the rod inside the cylinder at $1 \mathrm{GHz}$.

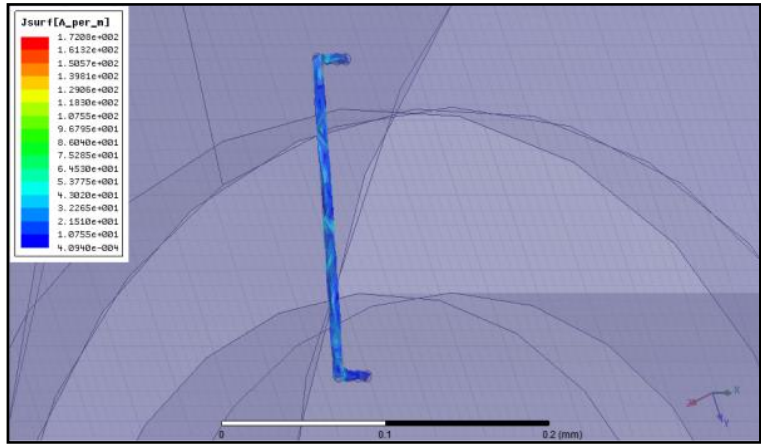

Fig. 4. Current density at surface of wire connecting rod and ring at $1 \mathrm{GHz}$

Grouping two, comprised of figures 5, 6 and 7, show the results of the model bombarded with a wave at $11 \mathrm{GHz}$. This shows that there is less E field enhancement at the external tip of the rod and more towards the interface of the rod and the cylinder, and that the concentration of the current density increases at the at the surface of the rod internal to the cylinder, and increases dramatically at the surface of the wire and the ring.

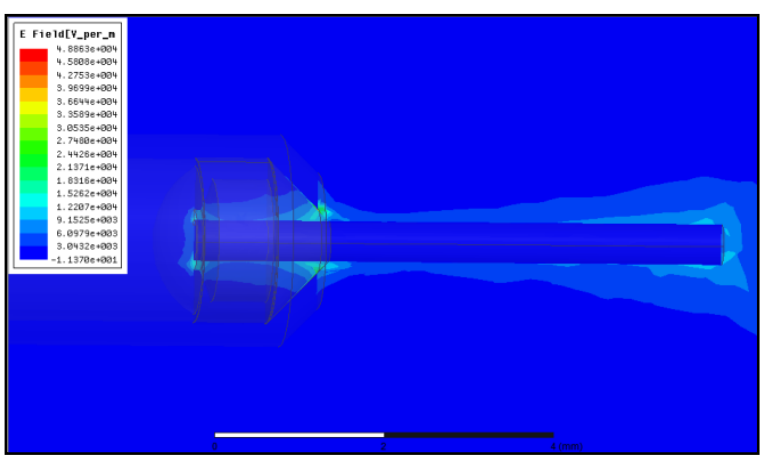

Fig. 5. E field results of $11 \mathrm{GHz}$ wave. 


\section{UNCLASSIFIED}

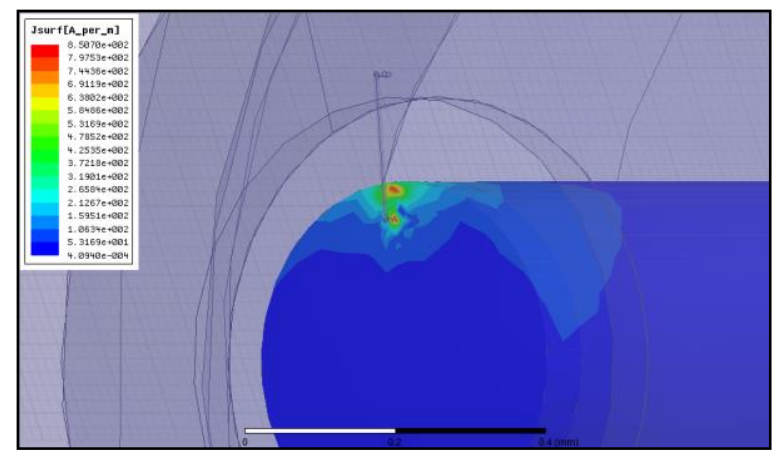

Fig. 6. Current density at surface of rod at $11 \mathrm{GHz}$

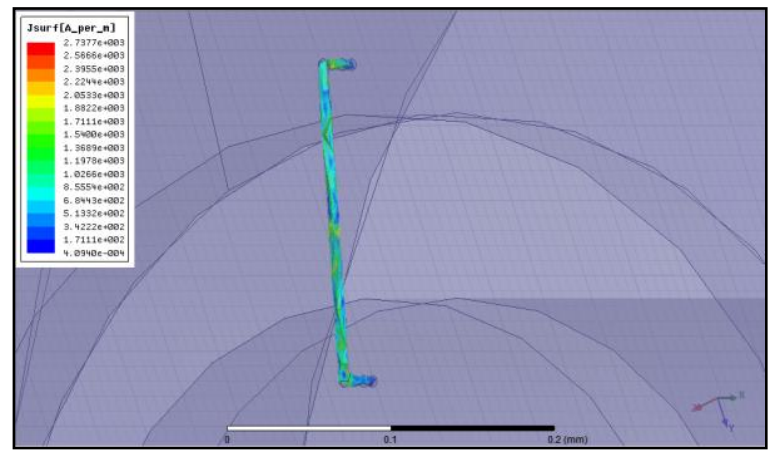

Fig. 7. Current density at surface of the wire connecting the rod and the ring at $11 \mathrm{GHz}$

Grouping three, comprised of figures 8, 9 and 10 , show the results of the model bombarded with a wave at $31 \mathrm{GHz}$. This shows that there is E field enhancement towards the interface of the rod and the cylinder, and that the concentration of the current density increases at the surface of the rod internal to the cylinder, and also at the surface of the wire and the ring.

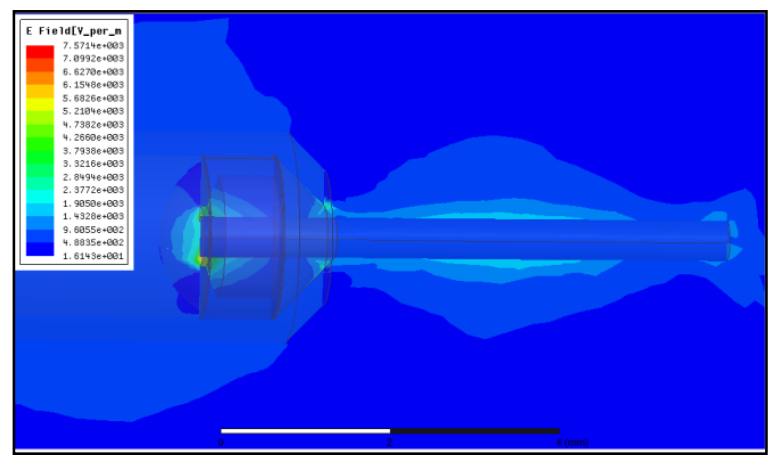

Fig. 8. E field results of $31 \mathrm{GHz}$ wave

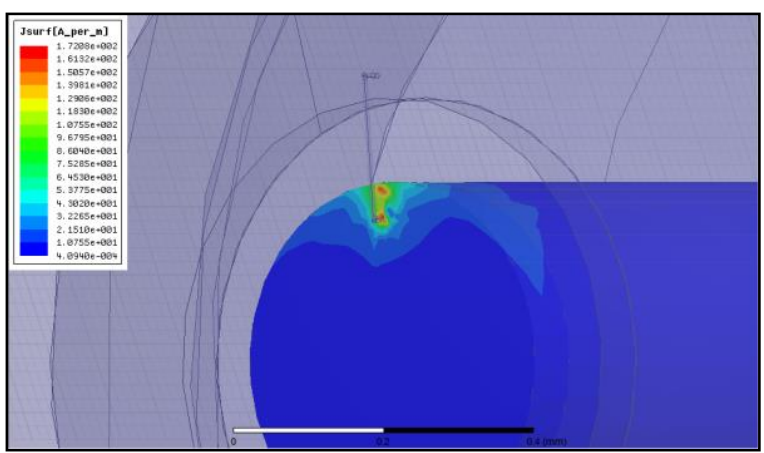

Fig. 9. Current density at surface of rod at $31 \mathrm{GHz}$.

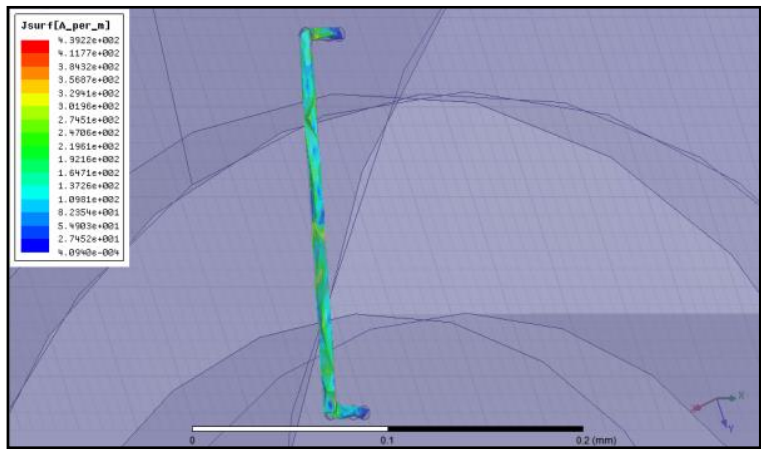

Fig. 10. Current density at surface of wire connecting rod and ring at $31 \mathrm{GHz}$

Collectively speaking at $1 \mathrm{GHz}$ the E field is focused on the end of the rod that is away from the other components. For 11 and $31 \mathrm{GHz}$ the E field is focused at the end of the rod that is connected to the rest of the components, which cause the higher surface current densities.

Fig. 11 quantitatively summarizes the simulation results. It reveals the key frequencies at which the greatest current density is induced throughout the multilayer system. The magnitude of the current density at the surface is shown for each object as depicted in Fig. 12-10.

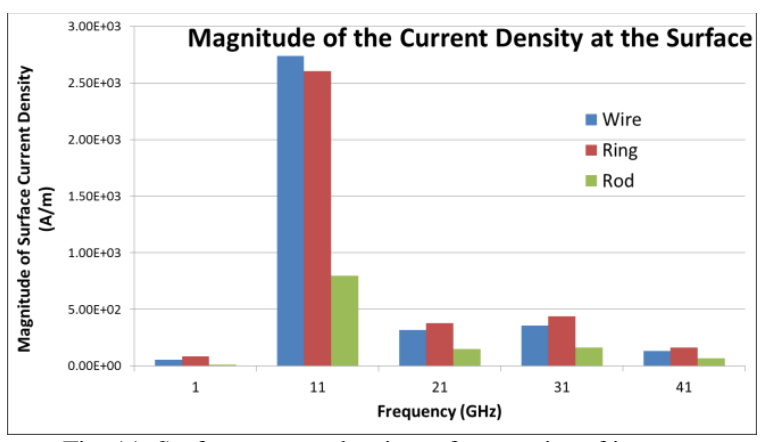

Fig. 11. Surface current density at frequencies of interest.

\section{Conclusion}

After a sweep of the frequencies from $1 \mathrm{GHz}$ to $101 \mathrm{GHz}$ the frequencies of interest are 1, 11, and 31

Distribution A/Approved for Public Release - PAO Log \# 122-14-25 FEB 2014/Distribution Unlimited 
GHz. These three frequencies impacted the system the most as shown from the E field and the current density plots. The E field is strongest at the tip of the rod away from the rest of the system at $1 \mathrm{GHz}$, but focuses towards the inside of the system at 11 and 31 $\mathrm{GHz}$. This is also apparent from the way the current density at the surface increases in the wire at 11 and $31 \mathrm{GHz}$.

$11 \mathrm{GHz}$ would affect this system the most since the current density at the surface of the wire and surrounding areas is highest at this frequency. It is possible that the wave is resonating with the small wire even though it is surrounded by other materials.

\section{FUTURE WORK}

Future work will include modification of the amplitude and include frequency diversity and repetition rate for EMP and REMP waves to determine how it influences the system. Also, the direction that the EMP is propagating towards the system will be changed.

\section{REFERENCES}

[1] E. S. William Radasky, "Intentional Electromagnetic Interference (IEMI) and Its Impact on the U.S. Power Grid," Metatech2010.

[2] ANSYS. (2012, February 10). ANSYS HFSS. Available: http://www.ansys.com/Products/Simulation+Tec hnology/Electromagnetics/High-

Performance+Electronic+Design/ANSYS+HFSS

[3] L. Thourel, The Antenna. Paris VI: Dunod, 1960.

Effort sponsored by the U.S. Government under Other Transaction number W15QKN-09-9-1001 between the National Warheads and Energetics Consortium and the Government. The US Government is authorized to reproduce and distribute reprints for Government purposes notwithstanding any copyright notation thereon. The views and conclusions contained herein are those of the authors and should not be interpreted as necessarily representing the official policies or endorsements, either expressed or implied, of the U.S. Government. 